\title{
Politik der Angst
}

\section{Die Regierung Berlusconi und die Ausländer}

\section{Immigranten in Italien und der Centrodestra}

Die Mitte-Rechts-Parteien haben in den letzten fünfzehn Jahren vor allem in Wahlkämpfen immer wieder das Gefühl der Angst und Verunsicherung thematisiert, das angeblich viele Italiener beherrscht. Dieses Gefühl habe, glaubt man den Medien, die dem Centrodestra nahestehen oder von Berlusconi kontrolliert werden, mit der massiven Präsenz illegaler Zuwanderer aus Nordafrika oder vom Balkan zu tun, die für Verbrechen und Gewalt verantwortlich seien.

Der vorliegende Aufsatz greift drei Fragen auf: Erlebte Italien wirklich eine Invasion von Immigranten? Wie haben die italienischen Regierungen in den letzten zehn Jahren auf das Problem der Einwanderung reagiert? Haben die Mitte-RechtsParteien das Thema Immigration für ihre Zwecke instrumentalisiert und, wenn ja, auf welche Weise? Um eine Antwort auf die erste Frage zu finden, werden Statistiken konsultiert, die von den Gewerkschaften und vom Innenministerium veröffentlicht wurden, während bei der zweiten Frage auf die Forschungsliteratur zurückgegriffen wird. Im Hinblick auf die dritte Frage gilt die Aufmerksamkeit vor allem der rechtskonservativen Presse, und zwar insbesondere der im Besitz der Familie Berlusconi befindlichen Zeitung „Il Giornale“. Wie hat sie einzelne Verbrechen, in die sogenannte Zigeuner verwickelt waren, präsentiert und kommentiert? Welche politischen Konsequenzen haben sich daraus ergeben?

Nach Angaben der katholischen Gewerkschaft Confederazione italiana sindacati lavoratori (CISL) gab es in Italien im April 2006 etwa 3,75 Millionen regulär gemeldete ausländische Bürger. Die Zahl der Illegalen schätzte man auf 750000 ; dazu gehörten auch diejenigen, die sich um eine offizielle Aufenthaltsgenehmigung bemühten. Unter den 3,75 Millionen Ausländern mit gültigen Papieren befinden sich 1350000 abhängig Beschäftigte, 30000 Handwerker, 30000 Händler, 170000 Landarbeiter, 8000 Bauern, 600000 Haushaltshilfen, 190000 Arbeitslose 
sowie 550000 Kinder und Jugendliche ${ }^{1}$. Der 2008 von der Caritas veröffentlichte 18. Einwandererbericht, der auf den Daten des Istituto nazionale di statistica beruht, meldete 3,43 Millionen Immigranten. Das entspricht 6,7 Prozent der Gesamtbevölkerung und liegt knapp über dem EU-Durchschnitt von 6,0 Prozent ${ }^{2}$. Mit Blick auf einzelne Herkunftsländer ergibt sich folgendes Bild: eine Million Rumänen, 402000 Albaner, 366000 Marokkaner. Diese drei Nationen liegen auch an der Spitze der Kriminalstatistik. Die Rumänen sind führend bei Totschlag, Sexualdelikten und Autodiebstählen, die Marokkaner bei Tätlichkeiten und Taschendiebstählen, während die Albaner bei Einbruchdiebstählen glänzen. In allen Fällen handelt es sich um Verbrechen, die die öffentliche Empfindsamkeit auf besondere Weise treffen und deshalb in starkem Maße zur Stigmatisierung der „Fremden“ als „Verbrecher“ beitragen. Der Bericht der Caritas spricht von einer „beachtlichen“ Ausländerpräsenz in Italien, die Jahr für Jahr zunehme. Außerdem verweist er auf die Tatsache, dass etwa die Hälfte der Ausländer Frauen seien, die mit ihren Familien in Italien lebten, was naturgemäß zu einer Stärkung der zweiten und dritten Immigrantengeneration führe. Schließlich erinnert der Caritas-Bericht daran, dass die Nachfrage nach ausländischen Arbeitskräften unverändert groß sei.

Aus all dem ergibt sich, dass die Gefahr einer besorgniserregenden Überfremdung in Italien nicht existiert. Es gibt keine Invasion von Ausländern, die Italienern die Arbeitsplätze streitig machen. Ebensowenig ist Italien als Grenzland ein Sonderfall, das den Einwandererfluten vom Balkan und aus Nordafrika in besonderer Weise ausgesetzt sei. Die Zahlen belegen vielmehr, dass Italien mit Blick auf die Ausländerpräsenz europäischer Durchschnitt ist und dass die weit überwiegende Mehrheit aus Familien mit Kindern besteht, die nichts anderes im Sinn haben, als hier bessere Lebensbedingungen zu finden.

\section{Gesetzliche Regelungen}

1998 verabschiedete die Regierung Prodi das Gesetz TurcoNapolitano. Sie antwortete damit auf das Bedürfnis nach größerer Sicherheit, das viele Bürger verspürten, und wollte insbeson-

${ }^{1}$ Vgl. Indici di integrazione degli immigrati in Italia, hrsg. vom Consiglio Nazionale dell'Economia e del Lavoro, Rom 2006.

2 Vgl. Immigrazione. Dossier statistico 2008, hrsg. von Caritas/Migrantes, Rom 2009. 
dere auf Forderungen eingehen, die von den unruhigen „Wählern der ökonomisch leistungsstärksten Regionen“ stammten ${ }^{3}$. Nach 1996 hatten sich vor allem in Mailand erste spontane „Bürgerwehren“ formiert, die ihre Stadt von Gammlern, Prostituierten und Dealern „säubern“ wollten und dabei von der Lega Nord unterstützt wurden.

Das Gesetz Turco-Napolitano brachte einige wichtige Neuerungen im Vergleich mit dem bis dahin geltenden, aus dem Jahr 1992 stammenden Gesetz Martelli: Es bestimmte, wie die Zahl der Arbeiter festgelegt wurde, die nach Italien einreisen durften; es schuf die Institution des "Sponsors“ - eine Person oder eine Einrichtung, die arbeitssuchenden Ausländern Unterkunft und Verdienst garantierte -, und es eröffnete Ausländern, die mindestens fünf Jahre in Italien lebten, die Möglichkeit, eine spezielle unbefristete Aufenthaltsgenehmigung zu beantragen, was sie von zeitraubenden bürokratischen Zumutungen befreite. Außerdem sah das neue Gesetz auch die Zurückweisung illegaler Einwanderer an der Grenze vor; politische Flüchtlinge waren davon freilich ausgenommen. Diese Bestimmung trat an die Stelle der Haft, die bis dahin bei illegalem Grenzübertritt verhängt worden war. Schließlich schuf das Gesetz neuartige Asylbewerberzentren, in denen Ausländer ohne Papiere maximal 30 Tage festgehalten werden konnten, um ihre Identität zu überprüfen.

Das Gesetz Turco-Napolitano ist - verglichen mit den entsprechenden Regelungen in anderen europäischen Ländern nicht besonders hart. So ist beispielsweise illegale Einwanderung, anders als in Frankreich, der Bundesrepublik, in Griechenland und Großbritannien, kein Straftatbestand. Hinzu kommt, dass Asylbewerber in anderen europäischen Ländern sehr viel länger in den auch dort bestehenden Zentren festgehalten werden können als in Italien.

2002 regelte die Regierung Berlusconi die Materie neu. Mit dem Gesetz Bossi-Fini wurde der digitale Fingerabdruck für diejenigen eingeführt, die eine Aufenthaltsgenehmigung beantragten; auch Abschiebung durch die Polizei ist seither möglich, und zwar in allen Fällen und nicht nur dann, wenn die Antragsteller für gefährlich gehalten wurden. Außerdem konnte die Aufenthaltsdauer in den Asylantenlagern auf bis zu 60 Tage ausgedehnt werden. Schließlich beseitigte das neue Gesetz die

${ }^{3}$ Marzio Barbagli, Immigrazione e sicurezza in Italia, Bologna 2008, S. 114 . 
Institution des „Sponsors“ und erschwerte damit die legale Zuwanderung von Arbeitssuchenden nach Italien.

Alles in allem unterscheidet sich das Gesetz Bossi-Fini nicht kategorial vom Gesetz Turco-Napolitano. Der Unterschied liegt in der Praxis, die unter den Mitte-Links-Regierungen deutlich liberaler war. Nach Ansicht der Soziologen Asher Colombo und Giuseppe Sciortino ist es neuerdings viel schwieriger, eine Aufenthaltsgenehmigung zu erhalten. Ferner ist die Zahl der Immigranten deutlich zurückgegangen, nachdem es in das Benehmen der Regierung gestellt wurde, die Quote der jährlichen Zuwanderer aus den verschiedenen Ländern festzulegen; die Praxis ist hier so restrektiv, dass nicht einmal der Arbeitskräftebedarf der Wirtschaft befriedigt werden kann ${ }^{4}$.

Das sogenannte Sicherheitsgesetz von 2009 hat die bürokratischen Hindernisse für Zuwanderer weiter erhöht. Die Aufenthaltsdauer in Asylantenlagern kann jetzt bis zu sechs Monate betragen. Außerdem wurde der Straftatbestand der Illegalität geschaffen, der jedoch nicht mit einer Haftstrafe bewehrt ist, sondern mit einer Geldstrafe und der Ausweisung. Um das älteste Gewerbe der Welt in den Griff zu bekommen, war ferner vorgesehen, auch die Kunden der Prostituierten zu bestrafen. Die Koalitionsparteien ließen diese Bestimmung aber schließlich wieder fallen.

\section{Das Beispiel der Rumänen}

Der eingangs erwähnte Caritas-Bericht bezieht sich auch auf die Frage, ob rumänische Immigranten tatsächlich eine so große Gefahr für die Sicherheit darstellten, wie häufig behauptet wurde. 1980 lebten etwa 8000 Rumänen in Italien, 17 Jahre später lag ihre Zahl mehr als hundert Mal so hoch. Anfang 2007 schätzte man, dass sich etwa 556000 Rumänen offiziell in Italien aufhielten; für 2008 ging man von 1016000 legal oder illegal in Italien lebenden Rumänen aus. Rund drei Viertel kamen auf der Suche nach Arbeit nach Italien, bei einem Viertel gab die Familienzusammenführung den Ausschlag. Mehr als die Hälfte (53,4 Prozent) der rumänischen Einwanderer sind Frauen.

Wer sich mit der rumänischen Immigration befasst, wird rasch mit dem Argument konfrontiert, dass die Ausländerkriminalität in Italien ein „ernstes Problem“ sei und dass überproportional

${ }^{4}$ Vgl. Asher Colombo/Giuseppe Sciortino, Gli immigrati in Italia, Bologna 2006, S. 68f. 
viele Rumänen in Delikte wie Totschlag, Sexualverbrechen, Autodiebstahl, Raub, Einbruchdiebstahl, Eigentumsdelikte, Erpressung verwickelt seien. Dazu kommt die Feststellung, rumänische Kriminelle hätten sich auf das Geschäft mit der Prostitution spezialisiert und verschleppten junge Frauen aus den ärmsten Regionen ihrer Heimat nach Italien; insgesamt sollen zwischen 18000 und 35000 Prostituierte aus Rumänien und aus anderen Ländern in Italien ihre Dienste anbieten.

Noch gravierender ist, dass ein Drittel der straffällig gewordenen Minderjährigen aus Rumänien stammt. 2004 waren es 4000, wobei die meisten Frauen sind, aus „Zigeuner“-Familien kommen und sich auf Eigentumsdelikte kapriziert haben; etwa 1000 Minderjährige wurden in Aufnahmelager gesteckt. Mit mehr als 2000 stellen die Rumänen auch die größte Gruppe unter den alleinlebenden Minderjährigen, die von ihren Eltern verlassen beziehungsweise verkauft wurden oder ausgerissen sind, um bedrückenden Familienverhältnissen zu entkommen.

Wenn in der italienischen Öffentlichkeit von den Rumänen die Rede ist, so bezeichnet man sie zumeist abwertend als zingari, also als Zigeuner. Beide Begriffe werden fast synonym gebraucht, was nicht zuletzt daran liegt, dass die meisten „Zigeuner" in Italien die rumänische Staatsbürgerschaft haben. Die Angst vor den zingari hat seit dem EU-Beitritt Rumäniens im Jahr 2007 beträchtlich zugenommen. Aus einer ISPO-Untersuchung, die der Soziologe Renato Mannheimer 2008 vorgelegt hat, geht hervor, dass 81 Prozent der Italiener zingari für „wenig oder gar nicht sympathisch" halten. Auf Platz zwei dieses Negativ-Rankings liegen die Albaner mit 74 Prozent, gefolgt von den Rumänen mit 64 Prozent, während 51 Prozent die Philippiner für sympathisch erklären ${ }^{5}$.

Alle diese Daten zeigen, dass in puncto Einwanderung kein Notstand herrscht. So gravierend die Probleme auch sein mögen, die mit den Rumänen und „Zigeunern“ in Italien zusammenhängen: Es gibt keine Wellen von Gewalt, die von Immigranten ausgelöst werden. Es bestehen aber nahezu ideale Voraussetzungen, die häufig in einen Topf geworfenen Rumänen und zingari zu Feinden zu stempeln, auf die sich der Hass der einheimischen Bevölkerung konzentrieren kann - und dafür, diese Gefühle und Ressentiments politisch zu instrumentalisieren.

Als Beispiel für diese politischen Strategien soll im Folgenden die Perzeption der „Zigeuner“ in der italienischen Gesell-

5 Vgl. Corriere della Sera vom 7.5.2009: „L'Italia che non ama i rom“. 
schaft analysiert werden, einer ethnischen Minderheit also, die sich schwer tut mit ihrer Umgebung - und es dieser schwer macht, weil sie aufgrund stark differierender Lebensstile und Realitätsdeutungen nicht leicht zu integrieren ist. Dabei wird gezeigt, wie die rechtsgerichtete Presse diese Gruppe beschreibt, die umstandslos als deviant und kriminell gebrandmarkt wird und selbst von denen nur schwer zu verteidigen ist, die ihr nicht a priori mit Ablehnung begegnen ${ }^{6}$.

\section{Der Fall Mailat}

Als Anfang November 2007 das Rennen um das Amt des Bürgermeisters von Rom eröffnet wurde, standen sich Francesco Rutelli von der Linken und Gianni Alemanno von der Rechten gegenüber. Rutelli, der dieses Amt bereits zwei Wahlperioden lang bekleidet hatte, galt als Exponent der Kontinuität, sollte er doch den scheidenden Bürgermeister Walter Veltroni ersetzen, der - wie Rutelli - dem linken Lager angehörte. Alemanno hingegen, zuvor Landwirtschaftsminister in der Regierung Berlusconi, versprach einen Neuanfang, der ganz im Zeichen dessen stehen sollte, was Berlusconi und seine Mitstreiter von der Lega Nord und der Alleanza Nazionale erstrebten.

Die Pressekampagne der Rechtsparteien war überaus aggressiv und bezog sich vor allem auf das Thema Sicherheit. Diese Akzentsetzung erlaubte es Alemanno und den Seinen, die Regierung Prodi wegen des angeblichen Missmanagements beim EUBeitritt Rumäniens am 1. Januar 2007 anzugreifen, der zur Invasion der zingari in Italien geführt habe, und sie eröffnete ihnen die Möglichkeit, die Stadtverwaltung Roms aufs Korn zu nehmen, die Dutzende von legalen oder illegalen „Zigeunerlagern" geduldet hatte. Das Fernsehen griff dieses Thema geradezu begierig auf, wie überhaupt gesagt werden muss, dass das Fernsehen in der Endphase der Regierung Prodi 2006/07 beträchtliche Teile seiner Sendezeit dem Thema Verbrechen und Kriminalität widmete. Nach einer Analyse der Radikalen Partei, die am 8. März 2009 in der Zeitung „La Repubblica“ zu lesen war, verdoppelte sich die Fernsehberichterstattung über Verbrechen von 2006 bis 2007. In den Nachrichtensendungen

${ }^{6}$ Vgl. Giorgio Bezzecchi/Maurizio Pagani/Tommaso Vitale, I rom e l'azione pubblica, Mailand 2008; Michele Mannoia, Zingari - che strano popolo! Rom 2007; Erica Rodari (Hrsg.), Rom, un popolo, Mailand 2008. 
des öffentlich-rechtlichen Fernsehens nahm dieser Komplex 2005 etwa 11,5 Prozent ein - 2007 belief er sich auf 22,3 Prozent, während im Privatfernsehen Berlusconis eine Ausweitung von 10,7 auf 22,6 Prozent zu konstatieren war.

Das war der Hintergrund des Falles Mailat, der von den Rechtsparteien nach Kräften instrumentalisiert wurde. Was war geschehen? Am 30. Oktober 2007 vergewaltigte und tötete Romulus Mailat die fast fünfzigjährige Giovanna Reggiani, die sich auf dem Weg vom Bahnhof zu ihrer Wohnung im Stadtteil Tor di Quinto befand. Das Verbrechen des Rumänen, der in einem illegalen Lager lebte, erfüllte die ganze Stadt mit Abscheu und Entsetzen. Opfer und Täter eigneten sich in geradezu idealer Weise, um von der politischen Rechten für ihre Zwecke ausgebeutet zu werden: Giovanna Reggiani, die gutbürgerliche Frau eines Marineoffiziers, war als Opfer wie geschaffen dafür, Identifikationsgefühle in der Mitte-Rechts-Wählerschaft zu wecken, während sich Romulus Mailat, ein „Asozialer“ aus einem Lager, gleichsam zum perfekten Monster stilisieren ließ, das die Ängste der rechten Wähler in urbanen Zentren evozierte. Auch das Viertel, in dem sich das Verbrechen ereignete, ordnete sich in dieses Schema ein: hier die feinen Villen der Offiziere, dort die Baracken eines Nomadenlagers als perfekter Kontrast zwischen dem Ideal wohlgeordneter Verhältnisse und dem Albtraum der dort lebenden Bürger, die auf eine fremde Gesellschaft ohne Regeln treffen und mit ihren Ängsten mitnichten allein stehen. Vergewaltigung und sexuelle Gewalt gegen Frauen sind allgemein geächtete Verbrechen, deren entrüstete Ablehnung die öffentliche Meinung jeglicher Couleur eint. Feministinnen und Neofaschisten stehen hier Seite an Seite.

Zur Speerspitze der Vorverurteilung und zu den Wortführern der Hasskampagne gegen die "Zigeuner“ entwickelte sich „Il Giornale“, die Zeitung der Familie Berlusconi, die im Wahlkampf den Kandidaten der Rechten, Alemanno, unterstützte. Am 3. November 2007 beschrieb sie das Verbrechen so:

„In Rom geht es vielleicht noch nicht so zu wie in den Ghettos von Soweto, ,wo die Frauen unter aller Augen vergewaltigt und umgebracht werden', wie Daniela Santanchè, eine Abgeordnete der Alleanza Nazionale, sagte. Der Vorfall von Tor di Quinto, der barbarische Angriff eines Rumänen auf Giovanna Reggiani, die im [Krankenhaus] Sant'Andrea um ihr Leben kämpft, scheint für unsere Stadt aber doch ein definitiver Wendepunkt zu sein. Nichts wird danach wie früher sein, die Stadt ist empört, und selbst Bürgermeister 
Walter Veltroni scheint es endlich zu begreifen, wenn er Ausweisungen fordert und damit die lang gehegte Utopie einer Aufnahmegesellschaft dementiert. Die Frauen haben Angst, die ganze Stadt hat Angst - vor allem in Tor di Quinto, in jenem geraden, langen und dunklen Sträßchen, das den Bahnhof an der Strecke Rom - Viterbo [...] mit der belebten Flaminia Vecchia verbindet." 7

Am 2. November besuchte ein Journalist von „Il Giornale“ Romulus Mailat im Gefängnis. Er schilderte den Häftling so:

„Wie sehen die Augen eines Monsters aus? Augen, in denen sich der Bodensatz der Menschlichkeit widerspiegelt, die Finsternis wilder Zeiten, die Gewalttätigkeit, die sich heute noch viel leichter ausleben lässt als in früheren, längst vergangenen Zeiten - um grundlos zu töten. Angst vermischt sich in den dunklen Augen des 24-jährigen, im Gefängnis Regina Coeli inhaftierten Rumänen mit Verwunderung - Augen, die ,Ruhe 'vortäuschen, um die Freiheit wiederzugewinnen, ohne viel dafür zu bezahlen. An den Händen sieht man einige Kratzwunden, die beeindrucken und anklagen sollen, auch wenn solche kleinen Verletzungen und Schürfwunden im Streunerleben der Zigeuner zum Alltag gehören. ${ }^{\text {" }}$

Die Tatsache, dass Mailat von einer „Zigeunerin“ angezeigt wurde, die im selben Lager wie der Täter lebte, fiel für die Mitarbeiter von „Il Giornale“ nicht ins Gewicht. Die mutige Anzeige, so hieß es in der Zeitung,

„passte nicht zum heruntergekommenen Milieu, in dem sich der brutale Angriff gegen Giovanna Reggiani ereignete: eine Zigeunerin, die das Gesetz der Omertà durchbrach und die Regeln ihres Clans missachtete, in dem sie die Polizei alarmierte und zur Baracke ihres Landsmannes führte, der gerade eine Frau fast zu Tode gebracht hatte, um ihr die Tasche zu rauben. In Wirklichkeit liegen die Dinge aber ganz anders: Emilia Neamtu hat erzählt, dass sie den 24jährigen Nicolae Romulus Mailat gesehen hat, wie er den blutüberströmten Körper der Reggiani weggezogen hat. Aber das ist nicht alles. Am Abend des vergangenen 30. Oktobers hat sie - ohne einen Finger zu rühren - zugesehen, wie die Frau des Kapitäns zur See, Giovanni Gumiero, ermordet

7 Il Giornale vom 1.11.2007: „Tor di Quinto ha paura: Orrore annunciato".

${ }^{8}$ Il Giornale vom 2.11.2007: „Il killer senza pietà: Ho solo rubato una borsa“. 
wurde. Mit dabei waren zwei weitere Personen - der Vater der Lebensgefährtin des Killers, Dorin Obedea, der Mailat geholfen hat, das Diebesgut zu verstecken, und Gherasim Neamtu, der Sohn derjenigen, die man bisher für die Superzeugin gehalten hat. Darüber hinaus haben zwei weitere Bewohner der Barackenstadt, die sich in der Nähe des Bahnhofs von Tor di Quinto [...] erhebt, wo die Reggiani angegriffen worden ist, daran mitgewirkt, Teile der Beute verschwinden zu lassen."

Anfang November erschienen im „Giornale“ die ersten Erklärungen von wichtigen Vertretern der italienischen Rechten:

„Forza Italia fordert den Rücktritt Walter Veltronis vom Amt des Bürgermeisters von Rom und schlägt in Erwartung von Neuwahlen vor, dem Präfekt von Rom, Carlo Mosca, die kommissarische Leitung zu übertragen. Die Blauen [Forza Italia] haben gestern Vormittag im Pressesaal des Abgeordnetenhauses eine dringliche Pressekonferenz angesetzt, weil sie, wie ihr stellvertretender Koordinator Fabrizio Cicchitto erklärt, ,die allzu vielen Lügen von Prodi und Veltroni über die Tragödie von Tor di Quinto satt haben'. Zum Beispiel, erläutert Cicchitto, ist es nicht wahr, wie sie sagen, dass die Regierung Berlusconi die Grenzen für so viele Verbrecher geöffnet hat. Das Gegenteil ist wahr. Alles nahm seinen Anfang mit Prodi, der die Invasion aus Rumänien nicht unterbunden hat. Von da an hat die rumänische Kriminalität die Hauptstadt überschwemmt - mit Raub, Vergewaltigung und tätlichen Angriffen. “10

Gianni Alemanno, der Kandidat der Mitte-Rechts-Parteien, versuchte, die Wasser der kollektiven Hysterie auf seine Mühlen zu leiten, während seinem Kontrahenten Rutelli vom Partito Democratico die Hände gebunden waren. Er konnte die Stadtverwaltung weder kritisieren, weil sie unter der Führung eines Parteifreunds stand, noch in Schutz nehmen, weil er genau wusste, dass sie nach den langen Jahren im Amt von vielen Römern für die Missstände verantwortlich gemacht wurde. Veltroni, der scheidende Bürgermeister, versuchte sich hingegen ebenfalls an die Spitze der gegen die Immigranten gerichteten Stimmung zu setzen:

9 Il Giornale vom 21.1.2008: „Uno massacrava Giovanna e noi ci spartivamo il bottino".

${ }^{10}$ Il Giornale vom 3.11.2008: „Forza Italia chiede le dimissioni di Veltroni“. 
„Ich wiederhole seit Monaten, dass der Massenzufluss an Einwanderern, der aus dem EU-Beitritt Rumäniens resultiert, mittlerweile eine andere Dimension gewonnen hat: Neben vielen anständigen Personen gibt es $\mathrm{zu}$ viele Kriminelle. Nicht ich sage es, sondern europäische Statistiken sagen es: Vor dem Januar 2007 war Rom die sicherste Großstadt der Welt. Aber jetzt ..."11

Im Wahlkampf schlug Rutelli die Bildung einer Kommission für Sicherheitsfragen und die Einführung eines elektronischen Armbands für Frauen vor, das sie in die Lage versetzen sollte, bei Überfällen Alarm zu schlagen. Alemanno ging einen Schritt weiter: Er verlangte die Ausweisung von 20000 straffällig gewordenen „Zigeunern“ und Ausländern, die Einsetzung eines staatlichen Sonderbevollmächtigten für Sicherheitsfragen und mehr Kompetenzen für die Stadtpolizei.

Und die Leser von „Il Giornale“? Wie reagierten sie und wie reagierten die Wähler der Rechten auf diese und andere Nachrichten ${ }^{12}$ ? Nachdem Mailat 2008 zu 29 Jahren Haft verurteilt worden war, herrschten Empörung und Verbitterung. Unter den vielen Kommentaren auf den Urteilsspruch fand sich mehr als einmal die Forderung nach lebenslänglich und der Todesstrafe:

„Warum nur 29 Jahre und nicht lebenslänglich? Dieser Mann (pardon: Hominide) hat eine ganze Familie zerstört, und in 15 Jahren wird er - als noch junger Mann - wieder frei sein. Verdammter Richter, der dieses Urteil gefällt hat.“

Ein anderer Leser nahm sich ebenfalls die Richter vor:

„Ihr Richter, die ihr vom Teufel abstammt. Eure Hermeline triefen von Blut. Für eure Ungerechtigkeit werdet ihr euch vor dem einzig wahren Richter verantworten müssen, und ihr werdet dort enden, wo Heulen und Zähneklappern herrschen. Die Schreie derjenigen, die ihr misshandelt und denen ihr Schmerzen und Demütigungen zugefügt habt, werden euch ewig verfolgen."

Bedauerlicherweise finden sich auf der Homepage von „Il Giornale" nicht alle Kommentare zu allen einschlägigen Artikeln; auch die ersten Reaktionen unmittelbar nach der Tat sind dort nicht eingestellt. Um eine Vorstellung von den spontanen Gemütsaufwallungen der Leser des „Giornale“ zu gewinnen,

11 Zit. nach Barbagli, Immigrazione e sicurezza, S. 138.

12 Die im Folgenden zitierten Kommentare finden sich in: www. ilgiornale.it. 
kann man einen anderen, besser dokumentierten Fall heranziehen, der sich im Mai 2008 in Ponticelli nahe Neapel zugetragen hat. Dort wurde eine zingara beschuldigt, bei einem Wohnungseinbruch ein kleines Kind geraubt zu haben. Die Reaktion der Menschen vor Ort bestand in einem Sturm auf einige Lager, wobei auch Molotowcocktails flogen. „Il Giornale“ tat sich wiederum unrühmlich hervor, indem das Blatt alle „Zigeuner“ als Kindesentführer denunzierte. Am 15. Mai 2008 konnte man in der Berlusconi-Zeitung lesen:

„Zigeunerlager dürfen nicht gestürmt werden. Punktum. Die Baracken der Zigeuner dürfen nicht in Brand gesteckt werden. Punktum. Das ist offensichtlich, banal und selbstverständlich, aber es ist gut, wenn man es mit allem Nachdruck bekräftigt. Wahr ist aber auch, dass man keine Kinder raubt, dass man sie nicht wie Sklaven behandelt und dass man nicht in Wohnungen einbricht. Aber auch hier sollte man so deutlich wie möglich werden: Das eben Gesagte ist keine Rechtfertigung oder kein mildernder Umstand für die Hitzköpfe von Ponticelli. Es ist aber ein Grund, um diejenigen anzuklagen, die es zugelassen haben, dass die Dinge so weit gedeihen konnten, und die sich heute nicht nur nicht mehr darum scheren, sondern die Leute auch noch unter Rassismusverdacht stellen, die die Zigeuner vor ihrer Haustür vertreiben wollen, obwohl sie doch verpflichtet wären, die Ursachen dieser Verbitterung zu verstehen und, wenn möglich, konkrete Antworten darauf zu geben. ${ }^{\text {13 }}$

Es ist nicht zu übersehen, dass hier die Absicht besteht, die Übeltäter von Ponticelli in Schutz zu nehmen. Und ebenso klar ist der Vorsatz, die Schuld an den Vorfällen der Regierung Prodi in die Schuhe zu schieben und Intoleranz und Rassenhass für politische Zwecke zu schüren. Instrumentalisierungen dieser Art sind kein italienischer Sonderfall, fremdenfeindliche und rassistische Ressentiments und Parteien gibt es überall in Europa. Ein staunenswertes Spezifikum ist aber, dass es nicht irgendeine Zeitung ist, sondern die Zeitung, die sich im Besitz der Familie des Ministerpräsidenten befindet, die rassistische Vorurteile bedient und gegen Minderheiten hetzt. Nicht weniger erstaunlich sind die Reaktionen der Leser, die den Sturm auf Zigeunerlager rechtfertigten und bejubelten - und die Vorfälle von Ponticelli so kommentierten:

13 Il Giornale vom15.5. 2008: „Vanno sgomberati“. 
„Ganz zugespitzt: Sehr gut gemacht, Neapolitaner! Eine weitere glorreiche Seite wie eure mythischen vier Tage [bei der Vertreibung der Deutschen 1943]. Wer sich verteidigt, verteidigt seine Würde.“

„Sie tun schon recht, wir sollten uns ein Beispiel nehmen und das Gleiche auch hier im Norden machen."

„10, 100, 1000 Brände! Um einen Satz zu zitieren, der den ehemaligen Abgeordneten Diliberto [von den Kommunisten] sehr freuen wird.“

„Lasst nicht locker, die Zündschnur brennt jetzt, vertreiben wir sie alle, raus aus Italien, sie kommen aus Rumänien, dorthin sollen sie zurückgehen, und wenn ihr Minister nach Italien kommt, lasst uns mit ihm sprechen. Man kann immer fragen, wer die Rechnung zahlt, wie viele Milliarden haben die von Steuern befreiten Zigeuner einkassiert? Ich, wir zahlen und werden von der letzten Regierung als Steuerhinterzieher hingestellt. Ich bin sehr verbittert.“

„Seit langer Zeit sind es die Italiener leid, von diesen Leuten ausgeplündert zu werden. Nach der versuchten Entführung eines Neugeborenen am letzten Samstag, war es unvermeidlich, dass so etwas passiert ist. Wenn man sich in die Lage derer versetzen würde, die ein weiteres Mal gelitten haben, kann man diese Handlungsweise nicht kritisieren. Glücklicherweise haben sich diese kommunistischen Gutmenschen etc. verzogen, die vom Geld der Italiener und nicht von ihrem eigenen lebten. Es lebe Italien, Grüße an alle.“

\section{Moralische Panik}

Der Migrationsexperte Marzio Barbagli sah die Sache so:

„Alle Politiker der Rechten, der Mitte und der Linken, alle oder fast alle Berichterstatter und Leitartikler [...] sind sich in einem Punkt einig: Der Mord an Giovanna Reggiani ist nicht denkbar ohne den EU-Beitritt von Rumänien und Bulgarien am 1. Januar 2007 und ohne das dabei erworbene Rechte der Bürger dieser beiden Staaten, sich in Italien und der übrigen EU frei zu bewegen."14

Die Statistik über Gewaltverbrechen und ihren Anstieg nach 2007 stützt diese These nicht. Die Visapflicht für Rumänien wurde bereits 2002 aufgehoben, so dass es zu einer enormen

${ }^{14}$ Barbagli, Immigrazione e sicurezza, S. 138. 
Zunahme der Zahl der Rumänen kam, die sich bis zu drei Jahren in Italien aufhalten durften. Ist deshalb der Schluss berechtigt, dass es im November und Dezember 2007 in Italien zu einer moralischen Panik kam, die sich im April 2008 wiederholte? Die Antwort lautet: ja. Diese Panik hat in entscheidendem Maße zum Sieg von Mitte-Rechts bei den römischen Kommunalwahlen von 2008 und zum Sturz anderer Mitte-Links-Stadtregierungen beigetragen. Aber damit nicht genug: Sie war ein maßgeblicher Faktor der Konsensbildung über eine Einwandererpolitik, die - stärker als früher - im Zeichen von Härte und Unnachgiebigkeit stehen sollte.

Aus dem Italienischen übersetzt von Thomas Schlemmer und Hans Woller. 